\title{
You foot the bill! Attacking NFC with passive relays
}

\author{
Yuyi Sun, Student Member, IEEE, Swarun Kumar, Member, IEEE, Shibo He, Senior Member, IEEE, \\ Jiming Chen, Fellow, IEEE, and Zhiguo Shi, Senior Member, IEEE
}

\begin{abstract}
Imagine when you line up in a store, the person in front of you can make you pay her bill by using a passive wearable device that forces a scan of your credit card without your awareness. An important assumption of today's Near-field Communication (NFC) enabled cards is the limited communication range between the commercial reader and the NFC cards - a distance below $5 \mathrm{~cm}$. Previous approaches to attacking this assumption effectively use mobile phones and active relays to enlarge the communication range, in order to attack the NFC cards. However, these approaches require a power supply at the adversary side, and can be easily localized when mobile phones or active relays transmit NFC signals.

We propose ReCoil, a system that uses wearable passive relays to attack NFC cards by expanding the communication range to 49.6 centimeters, a ten-fold improvement over its intended commercial distance. ReCoil is a magnetically coupled resonant wireless power transfer system, which optimizes the energy transfer by searching the optimal geometry parameters. Specifically, we first narrow down the feasible area reasonably and design the ReCoil-Ant Colony Algorithm such that the relays absorb the maximum energy from the reader. In order to reroute the signal to pass over the surface of human body, we then design a half waist band by carefully analyzing the impact of the distance and orientation between two coils on the mutual inductance. Then, three more coils are added to the system to keep enlarging the communication range. Finally, extensive experiment results validate our analysis, showing that our passive relays composed of common copper wires and tunable capacitors expand the range of NFC attacks to 49.6 centimeters.
\end{abstract}

Index Terms—Near-field communication, passive relay, magnetically coupled resonant wireless power transfer, attack.

\section{INTRODUCTION}

I MAGINE you are waiting in line at your favorite store, but unbeknownst to you, you just paid for the person in front of you. And, the person in front of you achieved this without any active electronics, making her attack virtually untraceable and (technically) FCC compliant [1]! Recent years have witnessed a proliferation of Near-field Communication (NFC) for secure and efficient communication in the Internet of Things. NFC works at the frequency $13.56 \mathrm{MHz}$ defined by the standard ISO/IEC $14443[2]$ and allows inexpensive battery-free tags to communicate wirelessly with powered readers over extremely short range ( 5 centimeters at most). In principle, NFC limits the maximum communication distance between the tag and reader to extremely close distances - an attractive security guarantee [3], [4]. Indeed, NFC has seen wide adoption for secure battery-free systems such as payment cards and identity (ID) cards.

However, recent studies have revealed several weaknesses of the NFC framework [5], [6], [7], [8], [9]. Malicious software on cellphones can exploit bugs in the Android NFC Stack or browser to attack NFC Services. For instance, infected phones close to contactless cards can surreptitiously read their information. More recent novel work has demonstrated using active relays to attack NFC cards [10], [11],

- Y. Sun, S. He, J. Chen and Z. Shi are with State Key Laboratory of Industrial Control Technology, Zhejiang University, Hangzhou, 310027, China, E-mail: yuyisun@zju.edu.cn, s18he@iipc.zju.edu.cn, cjm@zju.edu.cn, shizg@zju.edu.cn.

- S. Kumar is with the Department of Electrical and Computer Engineering, Carnegie Mellon University, Pittsburgh, PA, 15213, USA, E-mail: swarun@cmu.edu.

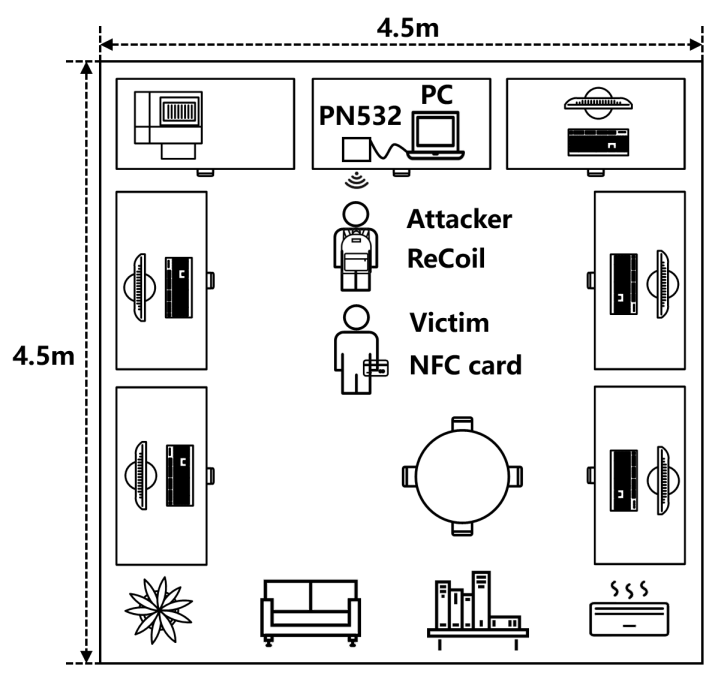

Fig. 1: The ReCoil scenario.

[12]. A smartphone or a circuit board with Android SDK API can serve as a man-in-the-middle, that relay signals from the NFC tag to the reader, violating the proximity assumption NFC communications.

In this paper, we build ReCoil - that, to our knowledge, provides the first completely passive attack on NFC applications. Passive relays are adopted due to the following reasons: 1) our device is totally composed of copper wires and capacitors, making the system a low-cost and lowconsumption one. 2) We design a wearable device which 
can be totally shielded by clothing or equipment that the attacker carries. Without power supply and active NFC reader, it is more possible for the attacker to attack the victim stealthily and secretly. 3) Since we do not revise the commercial protocols of NFC, our device is hard to detect. We allow NFC cards to be read at obover ten times their normal communication range using an array of passive relays that are thin, light-weight and small (readily shielded by clothing). These passive relays equipped with variable capacitors can steer near-field signal to provide maximized power to the NFC cards over further distances. A detailed experimental evaluation on commercial NFC readers and cards shows that we can relay query signals and responses between them over distances of 49.6 centimeters, under the environment shown as Fig. 1. against the advertised range limit of 5 centimeters.

Our core contribution is providing a near-field passive solution to maximize the output power to the NFC cards. Since the NFC tag is a purely batteryless device, ReCoil fully relies on the energy delivered wirelessly by a nearby NFC reader. Without additional power supply, it enlarges the communication range only by judiciously regulating the geometry parameters of the coils to obtain an optimized performance of the passive relays. This is complicated due to the fact that the distance and orientation among these coil relays can easily affect the mutual inductance between any two adjacent coils, resulting in a changed resonance frequency and downgraded performance. Therefore, it is important to optimize the design and geometry of the coils to maximize the energy transfer between the passive relays. ReCoil achieves this by carefully studying the inductance of the copper coils, through extensive modulations, simulations and experimentation. We first study varying shapes of the coils and show that square coils have better performance than other antennas. We then formulate an optimization problem to maximize the output power and compute the optimal parameters of the passive coils. Since non-linear solvers usually converge to sub-optimal local maxima, we design the ReCoil-Ant Colony Algorithm (RACA) to randomly initiate the beginning parameters and find the optimal geometry parameters which maximize the output power.

A second challenge that ReCoil addresses is to reroute the signal to pass over the surface of the human body because the signal is too weak to go through the human body directly. We build a Magnetically Coupled Resonant - Wireless Power Transfer (MCR-WPT) system to mitigate this challenge. Our design is a wearable waistband that surrounds the body and therefore mitigates its attenuation of the NFC signal when attacking the targetted NFC card. We add three more coils which are hidden in a backpack to keep the signal transmission. We then describe how the location of the coils can be optimized to maximize coupling between the NFC transmitter-receiver pair over extended distances. We further adapt the resonant frequency of the coils to the frequency of NFC signal by tuning the capacitor attached to the coils. A key challenge in ensuring the optimized energy transfer is the impact of objects between the relay and transmitter-received pair - the body and clothing of the attacker. When the attack device is attached on human body, the resonated frequency can be affected by compli- cated environment. Thus, we first perform experiments to shield coils from metallic materials on clothes by building a Ferrite sheet and model its impact. Further, our results in multipath-rich settings show that our system can still work at complicated environment in a robust manner.

We implement ReCoil as a light-weight multi-coil array that can be firmly worn on human bodies. We use Adafruit PN532 which has micropower as the commercial reader, making ReCoil a typical power-limited system. AWG 18 copper wires and variable capacitors are used to create the coils and apply intended phase shift. Based on the analysis and models, we use the High Frequency Structure Simulator (HFSS) to simulate and validate the performance of the passive coil relays by changing the corresponding orientation and separation. Based on these simulations and experimentation, we design the optimal dimension, distance and orientation of coils, achieving the optimal communication range. The results from our testbed show:

1) a distance of $49.6 \mathrm{~cm}$ in accessing other people's NFC cards, ten times that of the existing NFC systems.

2) robustness when attacking the NFC cards under multipath over a distance of $48 \mathrm{~cm}$.

3) successful attack over multiple NFC cards.

4) a practical deployment compatible with commercial NFC-based smartphones.

Contributions: We propose a novel system attacks the proximity-assumption of NFC using passive multi-coil relays. We design the optimal dimension, separation and orientation of these coils. Our system achieves extending the range of NFC cards successfully over distances of to $49.6 \mathrm{~cm}$ with negligible increase in latency.

The rest of the paper is organized as follows. In Sec. 2. we discuss related work on attacking NFC, passive relays design and how to detect potential attacks. NFC principles and the MCR-WPT model are introduced in Sec. 3 Attack model, key challenges and solutions are explained in Sec.4 4 Sec. 5 proposes our system design, mainly focusing on our algorithm and coil design. We implement ReCoil in Sec. 6 and conduct extensive evaluations in Sec. 7 Then, we discuss the limitations of our system and future work in Sec. 8 Sec. 9 concludes the paper.

\section{Related Work}

Active Attacks in NFC: Though NFC has limited communication range to guarantee the security of payments, there has been much work on active NFC relay hacking [13]. Much novel work increased the NFC range by the relay attack using smartphones [6], [14] or active relays [10], [11], [15] that amplify and forward NFC signals. Active NFC-enabled devices effectively increase the hacking range to 1.5 meters [16], with increased power consumption and hardware cost. Other work also focused on NFC eavesdropping [17], [18]. The eavesdropping range of magnetic field using passive NFC was first evaluated in [19], and a more powerful reader was designed to increase the communication range by $3-5$ times in [20].

Passive Attacks in NFC: Recent work on the design of passive attacks, primarily in the context of wireless power transfer, use the magneto-inductive (MI) principle [21], [22], [23], [24]. There has been much work using the MI model to 
improve the relay systems. Theoretical optimization problems for interference zero-forcing beamforming [25], [26] were formulated to analyze the optimal orientation and distance of relays. Binary load and frequency tuning [22] show an improvement of $35.6 \mathrm{~dB}$ in the whole passive relaying system. Lagrangian multi-user approach [21] was introduced and analyzed to obtain optimal orientations maximizing received power. Other work adopted passive relays in multiple applications. MagMIMO [27] designed a multiple-input multiple-output beamforming system and passive antennas attached to remote mobile phones, achieving a charging distance of 40 centimeters. MultiSpot [28] focused on multiple receivers, and beamformed the maximum current by inferring the number of receivers.

Detecting Attacks: Mechanisms of detecting the potential attacks on NFC have also been widely studied. Recent state-of-art work on detecting attacks in NFC fall into distance bounding [29], [30], [31]. A security framework of contactless access control $|31|$ considers the whole interaction between components, transforming the distance boundaries of access control to protect user privacy of NFC tags. Other work also used ambient sensors [32], [33], [34] and security middleware [35], [36], [37] to detect the malicious attack. Seven ambient sensors were used in [33] to separate normal and malicious transactions that may occur at the same time. A security middleware is designed in [35] to recognize CPU footprints with low latency less than a second. Further, infrared light was also adopted to prevent relay attacks in NFC [38].

Inspired by the models above, we build a practical system, ReCoil, that designs passive relays to break the guaranteed maximum communication distance. The total communication distance between the reader and the attacked card is enlarged to $49.6 \mathrm{~cm}$ using completely passive components, an improvement of $10 \times$ over commercial NFC.

Why Passive? Our system focuses on physical attack exploiting the design of completely passive relays. First, ReCoil does not consume power given that its relay coils are made from common copper wires and capacitors, making our system a low-cost and energy-efficient one. Second, our attack device can be relatively easily hidden without the power supply or the batteries, which means that the attacker can carries the attack device more stealthily when compared to the active ones. Then, though active transmitters bring us novel approaches to attack NFC, our system passively relays the query and authentication signals of the commercial reader over extended range without any active eavesdropping and data modification. Thus, it is relatively difficult to localize our relays. Furthermore, ReCoil tackles the complementary problem of aiming to enlarge the communication range of NFC cards without modifying the protocol. In the absence of active transmissions, modifications to the radio signal or changes to signal timing, these make ReCoil hard to detect using previous work on NFC threat mitigation.

\section{Preliminaries}

NFC has three modes of operation: the read/write mode, card emulation mode and peer-to-peer mode. In the $\mathrm{read} /$ write mode, an NFC device is used as a reader, communicating with the NFC tags. This mode is frequently used in access control system or mobile payments. In the card emulation mode, an NFC device acts as a passive card and can communicate with NFC readers when it receives enough energy to wake up. NFC smartphones operate in peer-to-peer mode to share useful information, like the contact lists or pictures between phones. In this paper, we consider NFC tags working in card emulation mode (e.g. payment cards or access cards) and a commercial reader transmitting signals to NFC tags.

The NFC Protocol for Authentication: When processing payments, an NFC reader keeps sending a query signal and waiting for the response from NFC cards. Once the NFC cards accumulate enough energy, they use load modulation to decode the reader's signal and then send a response which includes a 16-bit random number and an ID number (typically 4-7 bytes long). The payment process is authenticated after a successful handshake between the card and the reader.

Physics behind NFC: NFC tags use electromagnetic induction to supply power to wake up NFC tag chips. As Fig. 2 shows, the reader, powered by a power source $U_{0}$, transmits electric and magnetic energy through the coil antenna. The coil antenna on the NFC tag then observes an induced current and magnetic field due to mutual inductance. The NFC tag has a typical LC parallel circuit.

The larger the amount of magnetic flux from the transmitter's coil that goes through the receiver's coil, the larger the induced current is at the receiver. To maximize induced current, the coils are designed to be resonant at the NFC frequencies $(13.56 \mathrm{MHz})$ so that the inductance and capacitance: $j \omega L$ and $\frac{1}{j \omega C}$ cancel each other. We will find the optimal distance between two coils so as to maximize the mutual inductance between them.

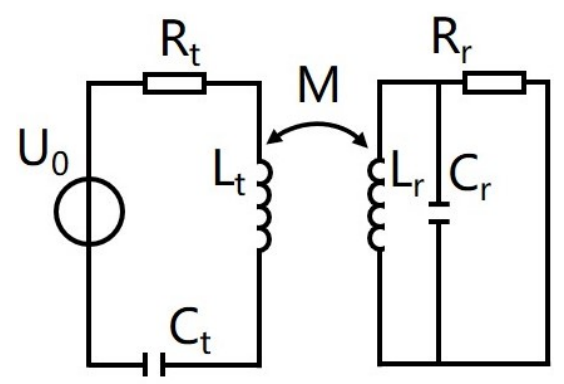

Fig. 2: Electromagnetic induction in NFC.

The net separation between a pair of coils for the same desired current at the receiver coil can be increased by using additional coil relays, which enhance the MCR-WPT. Passive relays help guide magnetic flux more efficiently from transmitter to receiver, allowing the receiver tag to receive the same current from a much longer distance (see Fig. 3). This model has an equivalent circuit to mathematically calculate the mutual inductance. After obtaining the mutual inductance, we can calculate the corresponding impedance which can represent the coupling degree between two coils.

The rest of this paper relies on these principles to study the effect of the human body, the coil design space (geometry, size, number of turns) as well as the circuit impedance 


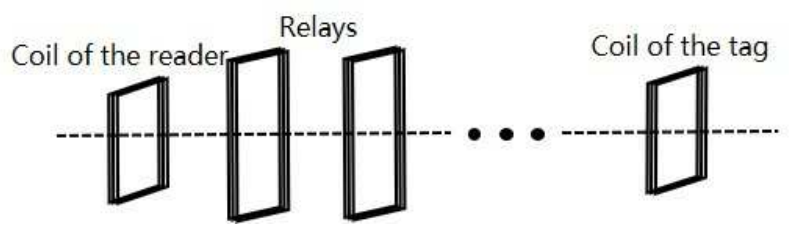

Fig. 3: The MCR-WPT model.

to maximize power transfer in real-world NFC passive manin-the-middle attacks.

\section{System MOdEL}

In this section, we introduce the attack model, and then explain the challenges and the solutions in our system. Our design in Sec.5 is around these key challenges.

\subsection{Attack Model}

As Fig. 4 shows, our attack model consists of a reader, an attacker equipped with the passive hacking device, and a victim who carries an NFC card. Our hacking device is composed of a half waist band and a backpack that contains three coils. The waist band consists of two square coils and HaBand. We use HaBand to reroute the signal pass over the surface of the human body, and use the square coils to focus the signal as much as possible. Then coils hidden in the backpack is used to convey the magnetic power to a further distance. When the attacker and the victim stand in the same line in a shop, the attacker can wear the waist band and the backpack to attack the NFC card of the person behind her.

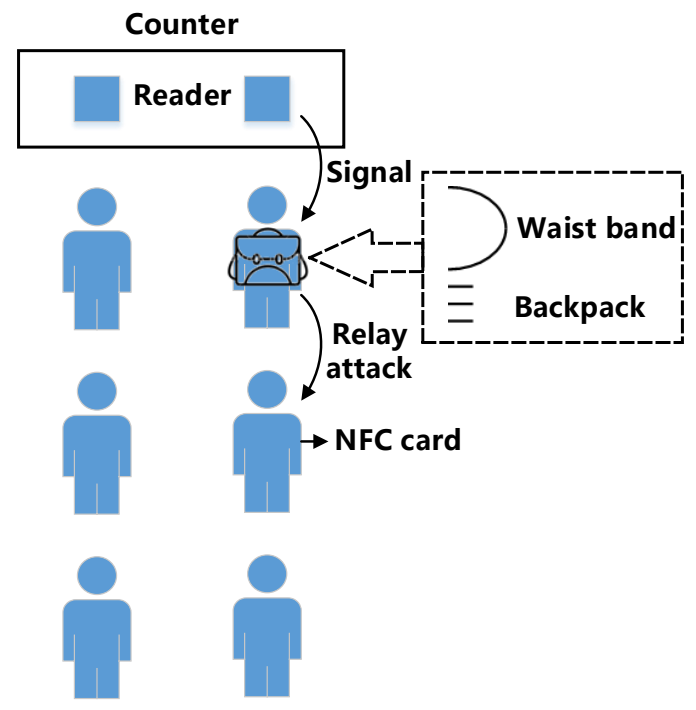

Fig. 4: The attack model.

\subsection{Challenges and Solutions}

Challenge 1: In our system, we aim to enlarge the communication range to attack the NFC cards by a wearable device. Our main goal is to transmit the signal of the reader to pass through the human body, and then to let the signal converge at the back of the attacker. However, in order to do so, we need to investigate how to determine the geometry parameters (size, number of turns, distance, orientation) that maximize the transmission range.

Solution 1: We formulate the problem to maximize the output power in relation to the geometry parameters of the relay coils. RACA is designed to find the optimum and inform our design. Based on the algorithm, we choose a square coil geometry that senses the magnetic field generated by the signal from the reader directly and maximizes signal power relayed.

Challenge 2: After we design the optimal parameters to relay the energy of the NFC reader, the fast-fading signal from the reader is attenuated as it is transmitted through these coils. In particular, the signal cannot pass through the human body directly merely with the help of coupling, resulting in a limited communication range. Further, we observe that the distance that the coils can enlarge is not proportional to the size of the relay coils. This brings about the challenging problem of how to reroute the signal to circumvent the human body and focus the magnetic power towards the back of the body to the maximum extent possible.

Solution 2: To address Challenge 2, we design HaBand, a big rectangular coil which is curved to fit the waist of a human body. Since the magnetic and electric energy is relayed, the signal is transmitted across HaBand towards the back of the body. Therefore, HaBand effectively reroutes the signal and most of the power to pass over the waist of the human body, while also weakening the magnetic field as little as possible. To improve range further, we build an MCR-WPT system, where we place one of the square coils in front of the human body to connect to HaBand and place another square coil on the back of the human body. In this way, the system can not only transmit the signal over the surface of the human body, but also focus maximum energy to the smaller coil on the back. In order to keep enlarging the communication range in a certain direction, we build a three-square-coil box which is hidden in a backpack based on RACA.

Sec.5 further elaborates the key challenges and solutions in the system design. Sec. 5.1 proposes the RACA and search the optimal parameters of the coils to solve Challenge 1. Sec. 5.2 shows how we combine the coils and focus the power to solve Challenge 2. Sec. 7 evaluates our design.

\section{System Design}

\subsection{Coil Design}

In this section, we first determine the shape of coils based on analysis and simulations. Then we formulate the energy transmission of the attack system, which can help us determine how to design the size and geometry of the passive relays. Next, we design the RACA to obtain the optimal geometry parameters.

\subsubsection{Shape Determination}

First, we compare the magnetic field strength generated by the coil with different shapes. We assume that all the types of coils are in the uniformly changing magnetic field, 


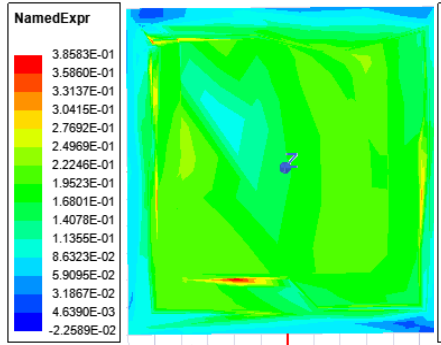

(a)

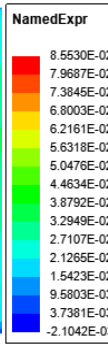

3.7381E-03 1042E-03

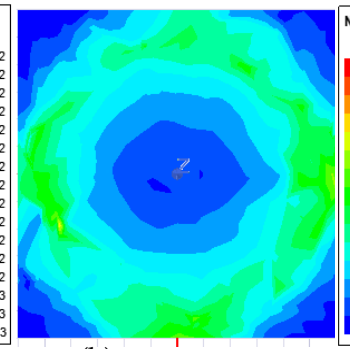

(b)

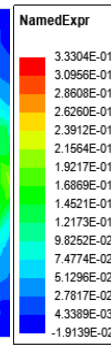

$-1.9139 \mathrm{E}-02$

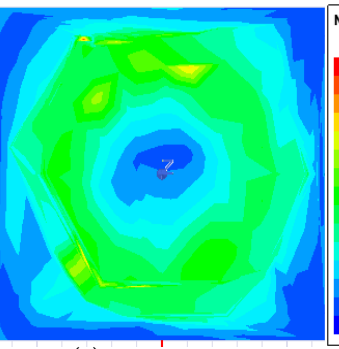

(c)

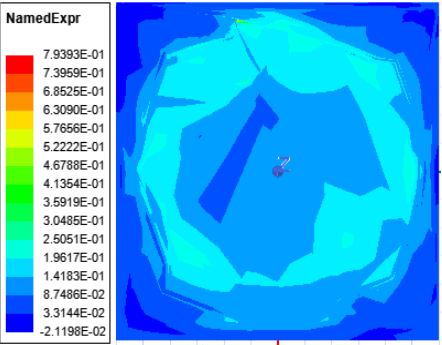

(d)

Fig. 5: The magnetic field of the: (a) square coil, (b) circular coil, (c) hexagonal coil, (d) octagonal coil.

and they have the same amount of magnetic flux (also the same area). We investigate square, circular, hexagonal and octagonal coils which are commonly used NFC antennas [39| to observe how they affect the mutual inductance. Based on their model of mutual inductance [40], [41], [42], though a circular coil has the maximum magnetic flux among the four types of coil, the energy transfer is related to the power source and is always case-by-case. Thus, we simulate the four coils in HFSS to investigate the magnetic field.

In order to simulate the reader PN532 (the commercial reader we use in Sec. 6 in HFSS, we build a signal source which has a rectangular coil antenna as PN532. Then, we draw a square, circular, hexagonal and octagonal coil respectively, and regulate them with the same parameters to observe the performance. The magnetic field relayed by these coils can be obtained, as Fig. 5 shows. We observe that the magnetic field relayed by the square coil is larger than that of the others. Therefore, square relay coils are adopted in our system design.

\subsubsection{Problem Formulation}

Wireless power transfer (WPT) is recently a popular technology which is widely used in wireless charging and other applications [43], [44], [45], [46]. Magnetically coupled inductive (MCI)-WPT and MCR-WPT are two typical techniques of WPT [47]. MCI-WPT has limited communication range, while MCR-WPT boosts longer distance with high transmission efficiency. Therefore, MCR-WPT helps us enlarge the communication range between the reader and the card effectively. An MCR-WPT system which has a serial circuit of transmitter, several serial circuits of resonate passive relays and a parallel circuit of receiver is defined as a Serial-Serial-Parallel (SSP) MCR-WPT system. Since the NFC card has a parallel circuit, the reader-relay-card system we design is an SSP MCR-WPT system, and the equivalent circuit is shown in Fig. 6

In the system, we assume that we need $n$ passive relays to enlarge the communication range. $I_{k}(k \in\{1,2, \cdots, n\})$ represents the current of relay $\# k$, shown in Eqn. $11 R_{k}$ represents the corresponding resistance, and $Z_{k}$ represents the equivalent impedance. Table 1 shows the symbols we define in ReCoil. $\omega$ is the angular frequency and $\omega=2 \pi f, f$ is the resonate frequency $(13.56 \mathrm{MHz})$ of NFC, $M_{12}$ represents the mutual inductance between relay \#1 and \#2, and so on. $M_{(i n)(1)}$ is the mutual inductance between the NFC reader and the relay \#1. $M_{(n)(o)}$ is the mutual inductance between relay \#n and the NFC card. The equivalent impedance due to the mutual inductance can be defined in Eqn. 2. where $Z_{i n}$ is the input impedance of NFC reader, $R_{o}$ is the output load of the NFC card.

$$
\begin{aligned}
& \left\{\begin{aligned}
I_{1} & =\frac{\omega^{2} M_{(i n)(1)} I_{i n}}{R_{1}+Z_{1}} \\
I_{2} & =\frac{\omega^{2} M_{12} I_{1}}{R_{2}+Z_{2}} \\
& \cdots \\
I_{n} & =\frac{\omega^{2} M_{(n-1)(n)} I_{n-1}}{R_{n}+Z_{n}} \\
I_{o} & =\frac{M_{(n)(o)} I_{n}}{L_{o}}
\end{aligned}\right. \\
& \left\{\begin{aligned}
Z_{n} & =\frac{M_{(n)(o)} R_{o}}{L_{o}^{2}} \\
Z_{n-1} & =\frac{\omega^{2} M_{(n-1)(n)}^{2}}{Z_{n}+R_{n}} \\
& \cdots \\
Z_{1} & =\frac{\omega^{2} M_{12}^{2}}{Z_{2}+R_{2}} \\
Z_{\text {in }} & =\frac{\omega^{2} M_{(i n)(1)}^{2}}{Z_{1}}
\end{aligned}\right.
\end{aligned}
$$

The output power $P_{o}$ is defined as

$$
P_{o}=I_{o}^{2} R_{o}
$$

where the $I_{o}^{2}$ is the current that passes through the load. The energy transmission efficiency can be defined as

$$
\eta=\frac{P_{o}}{P_{\text {in }}}
$$

where $P_{i n}=\left(Z_{i n}+L_{i n}\right) I_{i n}^{2}$.

Two coils that are put together is always with lateral, axial and angular misalignment [41]. The model of mutual inductance is $M=\frac{2 N_{1} N_{2} \mu_{0}}{\pi} \sqrt{S_{1} S_{2}} \int_{0}^{\pi} \frac{\left[\cos \theta-\frac{d}{S_{2}} \cos \phi\right] \Psi(k)}{k \sqrt{V^{3}}} d \phi$, where $S_{1}$ and $S_{2}$ represent half the side length of two adjacent square coils, $N_{1}$ and $N_{2}$ represent the number of turns of the two coils. $\alpha=\frac{S_{2}}{S_{1}}, \beta=\frac{c}{S_{1}}, \xi=\beta-\alpha \cos \phi \sin \theta$, $k^{2}=\frac{4 \alpha V}{(1+\alpha V)^{2}+\xi^{2}}, \Psi(k)=\left(1-\frac{k^{2}}{2}\right) K(k)-E(k)$, $V=\sqrt{1-\cos ^{2} \phi \sin ^{2} \theta-2 \frac{d}{R_{S}} \cos \phi \cos \theta+\frac{d^{2}}{S_{2}^{2}}}, K(k)=$ 


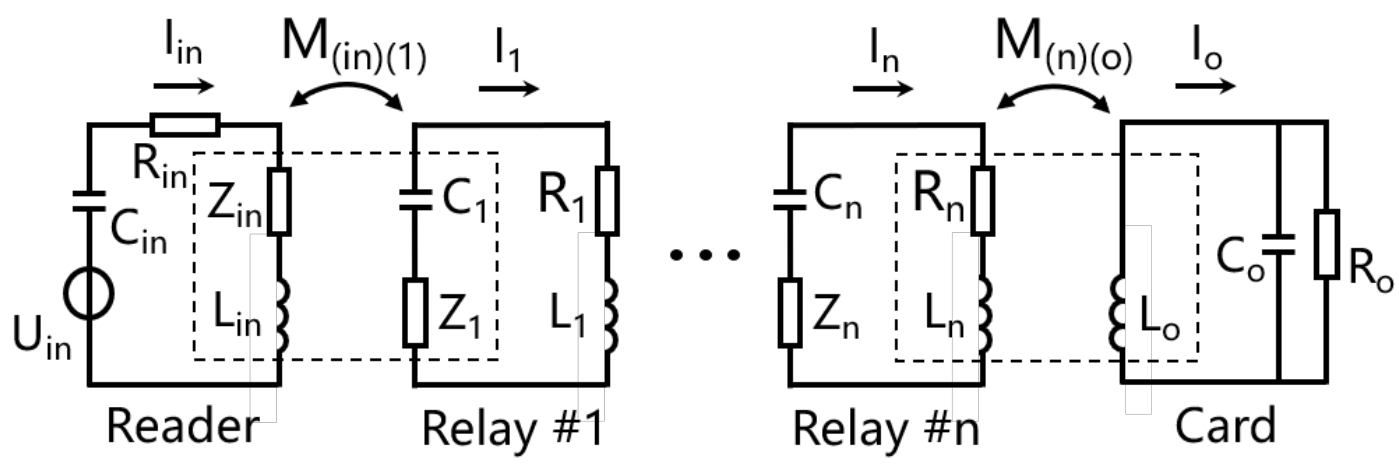

Fig. 6: The equivalent circuit of multiple coils in SSP MCR-WPT.

$\int_{0}^{\frac{\pi}{2}} \frac{1}{\sqrt{1-k^{2} \sin ^{2} \theta}} d \theta, E(k)=\int_{0}^{\frac{\pi}{2}} \sqrt{1-k^{2} \sin ^{2} \theta} d \theta$. We observe that the model of mutual inductance depends on the lateral misalignment $d$, the axial misalignment $c$, and the angular misalignment $\theta$. Next we use $S_{k}(k \in\{1,2, \cdots, n\})$ to represent the side length of relay $\# k$. $N_{k}$ is the corresponding number of turns. $S_{i n}$ is the side length of the NFC reader, and $S_{o}$ is the side length of the NFC card. $N_{i n}$ is the number of turns of the NFC reader, while $N_{o}$ is the number of turns of the NFC card. We define $d_{0}, \cdots, d_{n}$ respectively to represent the lateral misalignment between any adjacent two coils of the $n+2$ coils (NFC reader, NFC card, and $n$ relays). Besides, $c_{0}, \cdots, c_{n}$ and $\theta_{0}, \cdots, \theta_{n}$ also represent the corresponding axial and angular misalignment. We also define all the above symbols in Table 1

Based on the models above, in our problem, we maximize the output power of this coil system in Eqn. 5 below:

$$
\begin{gathered}
\max P_{o} \\
\text { s.t. }\left\{\begin{array}{l}
\eta^{n} \geqslant \eta_{0} \\
I_{i n}<I_{0} \\
S_{k} \leqslant S_{0}
\end{array}\right.
\end{gathered}
$$

According to Eqn. 6, in order to guarantee the signal transmission, the transmission efficiency $\eta^{n}$ when we use $n$ relays should be equal to or larger than the threshold $\eta_{0}$. Besides, due to the power constraint of FCC, the current of the NFC reader should be less than the threshold $I_{0}$. Further, due to the area constraint of human body, $S_{k}$ should be equal to or less than the threshold $S_{0}$.

This optimization problem is formulated as a nonlinear programming, which is non-convex and includes extensive nonlinear constraints. We cannot map our problem to an underlying convex problem and adopt convex optimization methods directly. Conducting the nonlinear programming solvers will bring about largely local optimum. Considering that parameter settings under experiments could be step by step, we try to analyze feasible regions and narrow them down as much as possible. Then we design RACA which will be introduced as follows to solve this problem.

\subsubsection{ReCoil-Ant Colony Algorithm}

Ant Colony Algorithm (ACA) is a heuristic algorithm that is inspired by the pheromone trail laying and following

\begin{tabular}{|c|c|}
\hline Symbol & Definition \\
\hline$n$ & The number of relays \\
\hline$k$ & Relay \#k $(k \in\{1,2, \cdots, n\})$ \\
\hline$I_{k}$ & The current of relay $\# k$ \\
\hline$I_{\text {in }}$ & The input current on the NFC reader \\
\hline$I_{o}$ & The output current on the NFC card \\
\hline$R_{k}$ & The resistance of relay $\# k$ \\
\hline$R_{o}$ & The output load of the NFC card \\
\hline$Z_{k}$ & The equivalent impedance of relay $\# k$ \\
\hline$Z_{\text {in }}$ & The input impedance of NFC reader \\
\hline$L_{\text {in }}$ & The inductance of the NFC reader \\
\hline$L_{o}$ & The inductance of the NFC card \\
\hline$N_{i n}$ & The number of turns of the NFC reader \\
\hline$N_{k}$ & The number of turns of relay \#k \\
\hline$N_{o}$ & The number of turns of the NFC card \\
\hline$S_{k}$ & The side length of relay $\# k$ \\
\hline$S_{\text {in }}$ & The side length of the NFC reader \\
\hline$S_{o}$ & The side length of the NFC card \\
\hline$f$ & The resonate frequency \\
\hline$\omega$ & The angular frequency $\omega=2 \pi f$ \\
\hline$M_{12}$ & The mutual inductance between relay \#1 and \#2 \\
\hline$M_{(i n)(1)}$ & $\begin{array}{l}\text { The mutual inductance between } \\
\text { the NFC reader and relay \#1 }\end{array}$ \\
\hline$M_{(n)(o)}$ & $\begin{array}{l}\text { The mutual inductance between } \\
\text { relay \#n and the NFC card }\end{array}$ \\
\hline$P_{\text {in }}$ & The input power \\
\hline$P_{o}$ & The output power \\
\hline$d_{0}, \cdots, d_{n}$ & The lateral misalignment between two adjacent coils \\
\hline$c_{0}, \cdots, c_{n}$ & The axial misalignment between two adjacent coils \\
\hline$\theta_{0}, \cdots, \theta_{n}$ & The angular misalignment between two adjacent coils \\
\hline$\eta^{n}$ & The transmission efficiency when we use $n$ relays \\
\hline
\end{tabular}

TABLE 1: Notations 
behavior of some ant species [48]. Artificial ants find the best path by sensing the density of the pheromone left by the ant colony. Inspired by this, we design RACA to obtain the optimal parameters that maximize the output power. We first divide the feasible areas into several scatter sets, narrowing down the feasible areas by taking account for real experiments. For example, we do not have to fix the iteration step $1 \mathrm{~mm}$, since $1 \mathrm{~mm}$ is negligible in real life. In order to reduce the computational complexity, $n$ should increase step by step, so we treat it as an initial parameter. In RACA, we initiate the corresponding geometric parameters, the number of iterations $T$, the volatilization coefficients $\rho$, the transition probability set $Q_{0}$ and the searching area set $\mathcal{A}$. Then, the randomly chosen parameters are input to calculate the initial ReCoil pheromone $\tau$ which describes the contribution to maximize the output power. The parameters with larger output power are given larger transition probability. We calculate the output power and update the parameters based on transition probability every loop. Algorithm 1 shows the details. $P_{o}^{n *}$ is defined as the maximum output power when we use $n$ relays $(n=1,2,3, \cdots)$, and we use $\eta^{n}$ to represent the transmission efficiency when we use $n$ relays. The local optimum can be avoided through $T$ iterations.

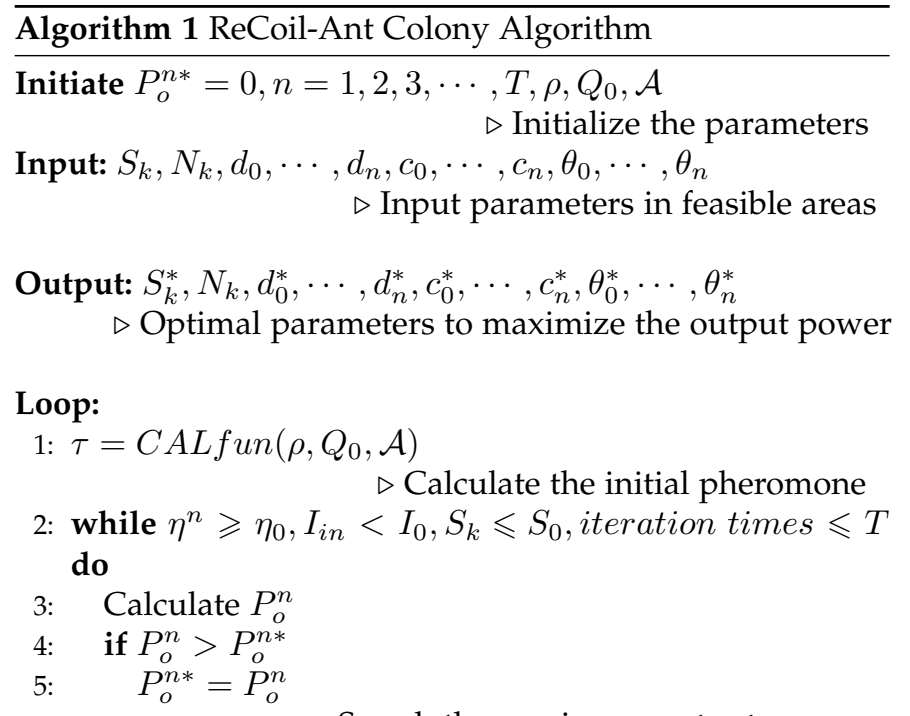

6: $\quad$ end if

7: $\quad$ Update $S_{k}, N_{k}, d_{0}, \cdots, d_{n}, c_{0}, \cdots, c_{n}, \theta_{0}, \cdots, \theta_{n}$

8: $\quad$ Update $\tau=(1-\rho) \times \tau+C A L f u n\left(\rho, Q_{0}, \mathcal{A}\right)$

9: end while

10: $S_{k}^{*} \leftarrow S_{k}$

11: $N_{k}^{*} \leftarrow N_{k}$

12: $d_{0}^{*}, \cdots, d_{n}^{*} \leftarrow d_{0}, \cdots, d_{n}$

13: $c_{0}^{*}, \cdots, c_{n}^{*} \leftarrow c_{0}, \cdots, c_{n}$

14: $\theta_{0}^{*}, \cdots, \theta_{n}^{*} \leftarrow \theta_{0}, \cdots, \theta_{n}$

We observe that the value of mutual inductance keeps dropping when the angular misalignment increases, thus when the two coils are parallel, the largest mutual inductance is obtained. Moreover, when the lateral misalignment increases, the mutual inductance between the two coils drops first and gradually increases to almost 0 . With the axial misalignment increases, the mutual inductance reduces

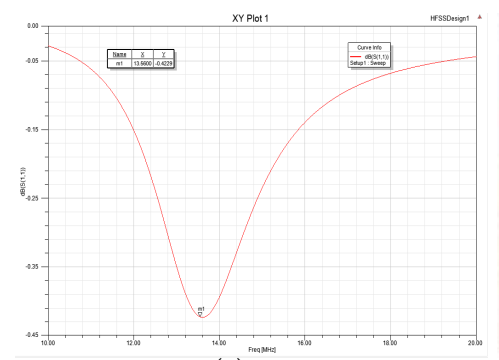

(a)

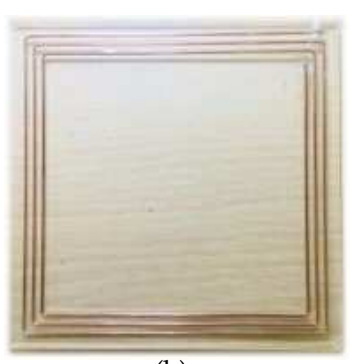

(b)
Fig. 7: (a) The return loss S11 under 51pF. (b) The prototype of the square coil.

and approaches to 0 .

We then tune the side length, the number of turns, the lateral, axial, and angular misalignment to study the output power. With the side length, the number of turns, or the axial misalignment of the square coils increasing, the output power increases first and then drops gradually. Further, the output power is inversely proportional to the lateral and angular misalignment. When the lateral misalignment is equal to 0 and the axial misalignment is equal to half the side length of the square coil, we have the largest output power. As a consequence, we build the square coils $10 \mathrm{~cm} \times 10 \mathrm{~cm}$ with three turns and a separation of two-millimeter between every two copper wires.

After we obtain the optimal parameters, we then build the model in HFSS and regulate the capacitor. We change the capacitor's capacitance progressively from $10 \mathrm{pF}$ to $90 \mathrm{pF}$. Initially, With the capacitance increasing, the return loss increases and has a peak at $51 \mathrm{pF}$. Then it gradually drops when the capacitance keeps increasing. Such a result validates that the coil can transmit the largest magnetic power at the resonant frequency $13.56 \mathrm{MHz}$, as Fig. 7(a) shows.

$$
f=\frac{1}{2 \pi \sqrt{L C}} .
$$

We design our prototype by using copper wires and capacitors, shown as Fig. 7(b). Based on Eqn. 7 and extensive experiments, we match a capacitance of $60 \mathrm{pF}$ for the system to achieve the largest distance expanded. Taking into account that the commercial capacitors have a 10\% error, the difference of results among the analysis, the simulation and the real coils can be acceptable.

\subsection{Coil Combination}

\subsubsection{HaBand Design}

Since the signal cannot go through the human body directly, we build HaBand which has a dimension of $10 \mathrm{~cm} \times 30 \mathrm{~cm}$. It also has three turns and a two-millimeter separation between every two copper wires. We match the appropriate capacitance to it based on Eqn. 7 Then, we change its curvature to match the curvature of human waist.

After we manually build the $10 \mathrm{~cm} \times 10 \mathrm{~cm}$ square coils and the $10 \mathrm{~cm} \times 30 \mathrm{~cm}$ HaBand, we combine them and build an MCR-WPT system, focusing the signal at the back of the human body as much as possible. When two coils 
horizontally laying in the same plane couple each other too close, one of them is the source of interference to the other, weakening the whole magnetic fields. When we gradually separate the two coils in the same plane, the coupled magnetic field would increase first and then drop. The parameters that describe the combined magnitude of the magnectic field in HFSS also validate this observation. We also evaluate this result by using Universal Software Radio Peripheral (USRP) in Sec.77 Indeed, this is the reason why we need a small square coil to absorb the energy from the NFC reader, before we use HaBand and another small square coil. Together, our approach focuses the signal and magnetic power in the desired direction around the attacker's body.

In order to obtain the whole optimization in our system, it is important to make sure that every coil has the best performance. Considering that there are some errors among different coils and different capacitors, we use the tunable capacitors which can be effortlessly hand-tuned to be matched to every unique coil.

\subsubsection{Backpack design}

After converging the signal at the back of the human body and regulating the optimal value of capacitors, we want to find the best way to keep the signal transmitting farther. Thus more coils need to be added to this system. As we know from Sec. 5.1. when we gradually separate two coils, the magnetic power increases first and reaches a peak, then it finally drops. We add three more coils based on the optimal distance between two coils we obtain. We also test the performance by changing the distance in HFSS. The results show that the two-coil system has a better performance when the distance is between $5 \mathrm{~cm}$ and $6 \mathrm{~cm}$, which validates the analysis in Sec. 5.1. In Sec.7, we introduce how we evaluate this range improvement using a software radio.

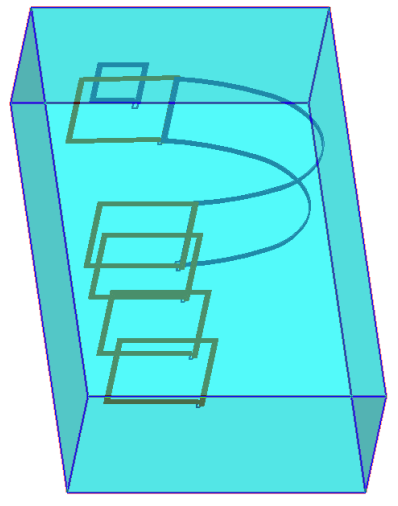

Fig. 8: The ReCoil simulation model in HFSS.

Orientation can also impacts the generated magnetic field. In order to verify the optimal orientation of the coils that we put in the backpack, we change the orientation of the coils in HFSS model. The system is expected to get the optimal performance when the two coils totally face each other. This result is consistent with that in Sec. 5.1. We let the distance between the two coils be $5 \mathrm{~cm}$ and change the orientation parameter of the second coil from 0 deg to $180 \mathrm{deg}$. When the orientation parameter is $0 \mathrm{deg}$, the system can generate the largest magnetic field. In Sec. 7 we also use USRP to evaluate our system performance by changing the orientation of coils. After we obtain the optimal distance between the coils and the orientation of the coils, a backpack is used to hide the additional three coils. As a consequence, more distance enlargement to hack people's cards can be achieved.

Based on the results above, we build our attack system in HFSS to observe the transmission process of the magnetic field. As Fig. 8 shows, the small rectangle in front of the whole model acts as the commercial reader which generates magnetic signals. Then, we introduce our system which consists of the square coils and HaBand. Another three square coils hidden in the backpack keep delivering the signal and energy far away from the band. We introduce several cross-sectional planes across the model to see the transmission process of the magnetic power. Column(a) in Fig. 9 illustrates the magnetic field around the square coil beside the reader. Column(b) introduces that the magnetic field generated by the square coil is gradually weaker and largely transmitted on HaBand. From Column(c), the magnetic field begins to converge on the square coils behind HaBand. In summary, the magnetic field is first absorbed by the square coil in front of the human body, and then it is transmitted by HaBand. It finally converges towards the coils in the backpack. These results validate our analysis from before.

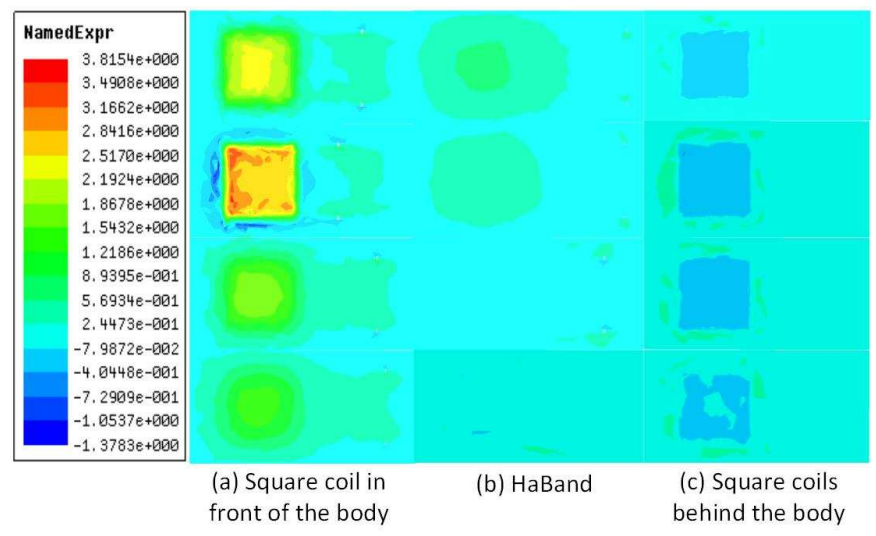

Fig. 9: The cross-sectional planes of the magnetic field in ReCoil system.

\section{IMPLEMENTATION}

We build the testbed that consists of a reader, an attacker equipped with ReCoil, and a victim who carries an NFC card. Our testbed is built in a $4.5 \mathrm{~m} \times 4.5 \mathrm{~m}$ office area, shown as Fig. 1. We place the PN532 NFC reader which is connected to a personal computer (PC) through the serial port on the desk. PN532 NFC shield is attached to Arduino through $\mathrm{I}^{2} \mathrm{C}$ interface. One person acts as the attacker, and the other who holds NFC cards acts as the victim. The waist band is worn on the attacker's waist and the three-coil box is hidden in a backpack. Through the serial port software, the PC on the desk reads the information about the hacked card, including its unique ID and whether it can be authenticated or not. 
Fig. 10 shows the design of the attack device. It is composed of the waist band and the three-coil box placed into the backpack. All the coils are fixed on acrylic plates which do not introduce interference. HaBand has a dimension of $10 \mathrm{~cm} \times 30 \mathrm{~cm}$, while the square coils have a dimension of $10 \mathrm{~cm} \times 10 \mathrm{~cm}$. We make HaBand curved by hot-melt technique to fit around the human body's waist. In order to guarantee the largest magnetic field, we regulate every tunable capacitors to achieve the best performance of the device. Fig. 10(b) also shows our design on the box we put into the the backpack. From the results in Sec. 5 , we first set the distance of every two of the square coils $5 \mathrm{~cm}$. Then, we make every two of them totally face each other, i.e., we set the orientation $0 \mathrm{deg}$.

In our system, we conduct five types of experiments. (1) Experiments of two-coil system. We build two square coils by using copper wires and capacitors. In this two-coil system, we deploy USRP to catch the received power. The distance between the two coils, the distance between the NFC tag and the last coil, and the orientation of the coils are changed during the process. We compare the results and optimize the parameters. (2) Experiments of the number of coils. We arrange the centroid of the square coils be in a straight line (like dominoes), and investigate the farthest distance that our system can enlarge by simply adding more square coils to our system. (3) Impacts of human body. We deploy our whole testbed on a human body to find the impact of the human body on the received power. We then deploy the Ferrite board on the human body to investigate the impacts on the whole distance. (4) Experiments amidst multipath. We evaluate the impacts of clothing and the impacts of multiple cards on the performance. Besides, considering the interference from the moving people, we add interference from iron plates, mobile phones and keys to our system to test the robustness of ReCoil. (5) Experiments on commercial smartphones. We adopt commercial NFCbased smartphones as the commercial readers to test the feasibility of our system.

\section{Evaluation}

In this section, we use USRP to validate the results we obtain in Sec. 5 and Sec. 6 We use the USRP with the DLP radio frequency identification (RFID) antenna to listen to the real signal from the reader PN532. The DLP RFID antenna also works at a frequency of $13.56 \mathrm{MHz}$. This USRP system provides us the received normalized voltage to describe the energy transfer. We first use these devices to listen to the signal generated by the reader and the response from the NFC card. We find that when the normalized voltage is larger than 0.22 , the card can always decode the query signal from the reader and send the response.

\subsection{Two-coil System}

We let two coils totally face each other and change the distance between them. We bind the DLP RFID antenna and the second coil together, minimizing the distance between the DLP RFID antenna and the second coil. From the results in Fig. 11. when the distance between the two coils is around $5 \mathrm{~cm}$ (half the side length of the coils), the normalized

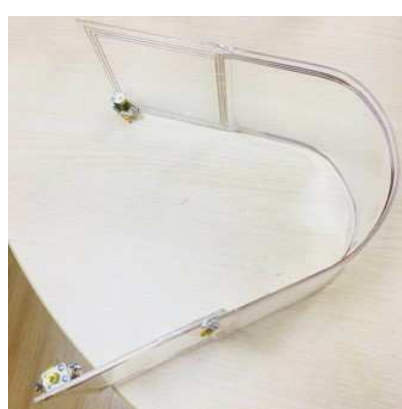

(a)

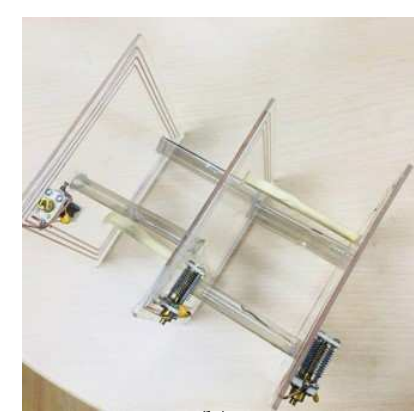

(b)
Fig. 10: (a) The waist band. (b) The three coils put into the backpack.

voltage received by the DLP RFID antenna reaches the peak, which means that the second coil can relay the largest power when the distance is $5 \mathrm{~cm}$. The result is consistent with that of the analysis and simulation. We observe that the farthest communication distance is $16 \mathrm{~cm}$ in Fig. 11 Further, we fix the distance between the two coils $16 \mathrm{~cm}$, and use the DLP RFID antenna as an NFC card. We slowly move the DLP RFID antenna far away from the second coil. As Fig. 12 shows, when the card moves, the received power reaches a peak at the distance of $4 \mathrm{~cm}$, then it begins to decrease. That is to say, the second coil cuts the maximum magnetic lines and generates the maximum magnetic field at $4 \mathrm{~cm}$. We observe that the error of normalized voltage in Fig. 11 is larger than that Fig. 12 This stems from the reason that the DLP RFID antenna is being too close to the second relay coil in Fig. 11 and brings more interference to our system. When we fix the distance between the two coils $16 \mathrm{~cm}$, we add the third relay coil to this two-coil system and find that the NFC card cannot respond to the reader anymore. This verifies our analysis that the distance between two adjacent coils should be fixed to maximize the power transfer.

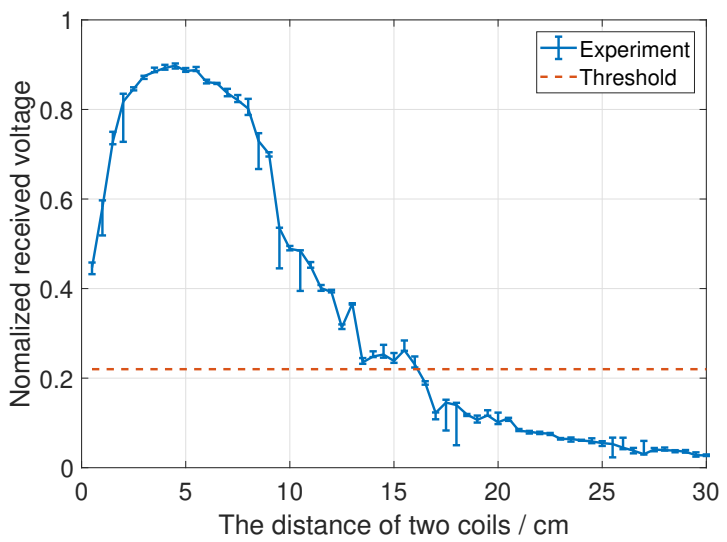

Fig. 11: The received voltage vs. the distance between two coils.

We then place two coils horizontally in the same plane, and we fix the distance between the card and the coils to $4 \mathrm{~cm}$. We progressively move one of the coils horizontally in the plane and observe the received normalized voltage. Shown as Fig. 13. when the $10 \mathrm{~cm}$ side of the two coils are 


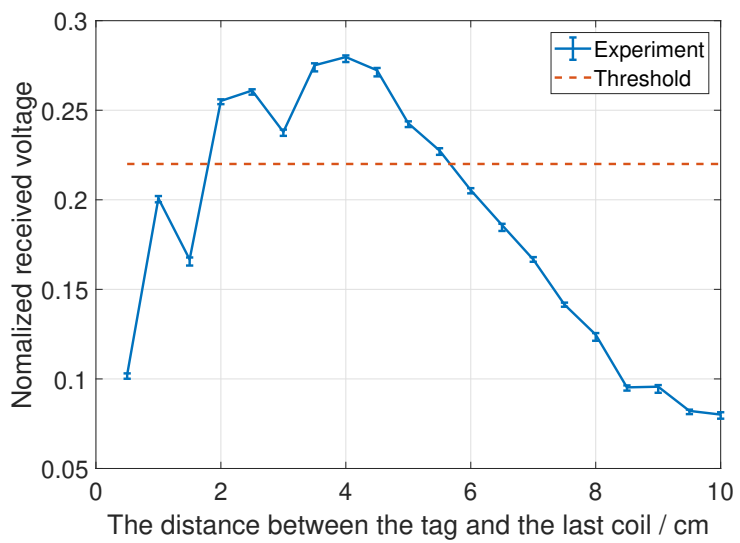

Fig. 12: The received voltage vs. the distance between the tag and the last coil.

precisely next to each other, we define the distance as $0 \mathrm{~cm}$. The distance is positive when the two coils become apart, and the distance is negative when they overlap. When the distance between the two sides of the coils is $0 \mathrm{~cm}$, the system has the best performance. This is the reason why we compactly attach the square coils and HaBand.

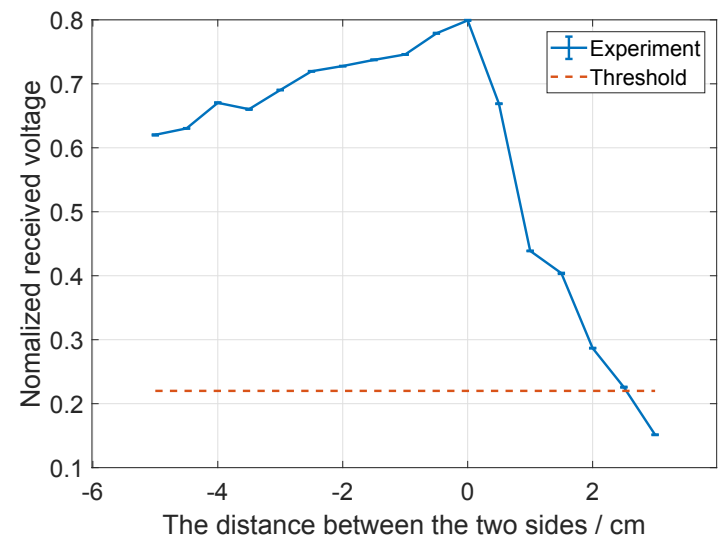

Fig. 13: The received voltage vs. the distance between the two sides of the coils.

In order to evaluate the orientation (angular misalignment) of the coils, we change the orientation of one of the two coils. Considering that we need enough space to rotate the second coil, we set a distance of $18 \mathrm{~cm}$ between the first coil and the second coil. When the two coils are totally parallel, we define the orientation of the second coil as $0 \mathrm{deg}$. When we rotate the second coil counterclockwise, the orientation is defined to be negative. The orientation is defined to be positive when the second coil is rotated clockwise. As Fig. 14 shows, the received voltage has a peak at the orientation of $0 \mathrm{deg}$. This means that we have the maximum mutual inductance at $0 \mathrm{deg}$, which validates the results in Sec. 5 The error shown in Fig. 14 is within $10^{-4}$. We observe that there are two valleys in Fig. 14 Since we do not consider the dynamic variability of the magnetic flux in the analysis, the second coil cuts the minimum magnetic field lines under $-70 \mathrm{deg}$ or $70 \mathrm{deg}$ in the real experiment.
Then the second coil cuts more magnetic field lines, making the energy increase when the angular misalignment is larger than 70 deg or less than $-70 \mathrm{deg}$.

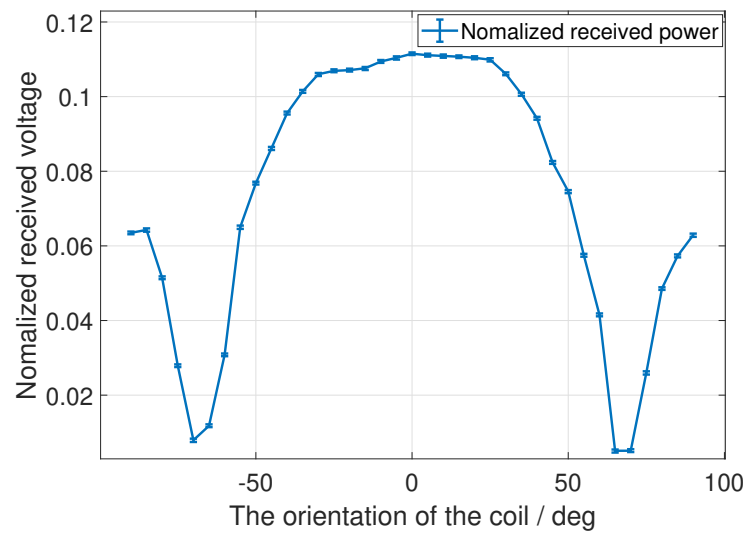

Fig. 14: The received voltage vs. the orientation of the coils.

\subsection{The Number of coils}

Then, we evaluate that how the number of coils impacts the attack distance when we arrange the centroid of the relay coils to be in a straight line (like dominoes). We deploy every two of the coils with a distance of $5 \mathrm{~cm}$, because the NFC card receives the largest energy under the circumstances. Then, we fix a distance of $4 \mathrm{~cm}$ between the reader and the first relay coil, and a distance of $4 \mathrm{~cm}$ between the NFC card and the last coil. As Fig. 15 shows, the communication distance rises to $54 \mathrm{~cm}$ till we have 11 coils. We observe once the number of coils is larger than 11, the NFC card cannot wake up and respond anymore. Thus, a distance of $54 \mathrm{~cm}$ can be obtained when 11 coils are arranged in a straight line. Since we obtain the best distance between two adjacent coils to maximize the output power, we keep adding the coils every $5 \mathrm{~cm}$. As we know from Sec. 7.1. when we keep the maximum communication distance every time we add a new coil, the signal stops at the third coil. Therefore, we do not select the maximum communication range every time. This is the reason why the curve in Fig. 15 is almost linear before the eleventh coil is added. However, the energy is attenuated during the process of adding more coils. After we add the twelfth coil, the NFC card does not respond anymore.

\subsection{Human Body}

We evaluate the distance between the NFC card and the backpack when we add the three-coil box to the system, shown as Fig. 16. We find that the distance that the device worn on human body can reach is $7 \mathrm{~cm}$, while the distance of the device without human body is $8 \mathrm{~cm}$. From the beginning, the energy around human body keeps dropping, this stems from the reason that the human body changes the energy transfer and as a result, changes the position of the maximum power. Then, the received voltage with human body is generally smaller than that without human body. Therefore, human bodies have the impact on the 


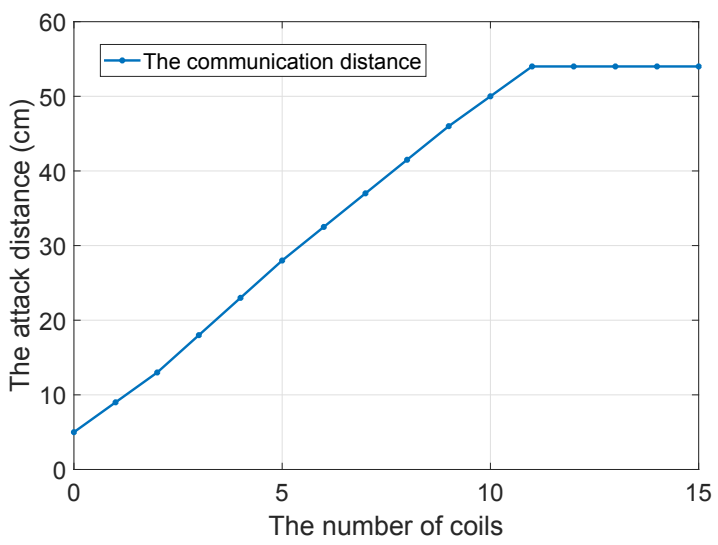

Fig. 15: The communication distance vs. the number of coils.

performance of the whole system, impairing the attack distance and moving the maximum point of received power. Compared to the communication range of existing NFC systems, our system can still achieve a large enlargement in range.

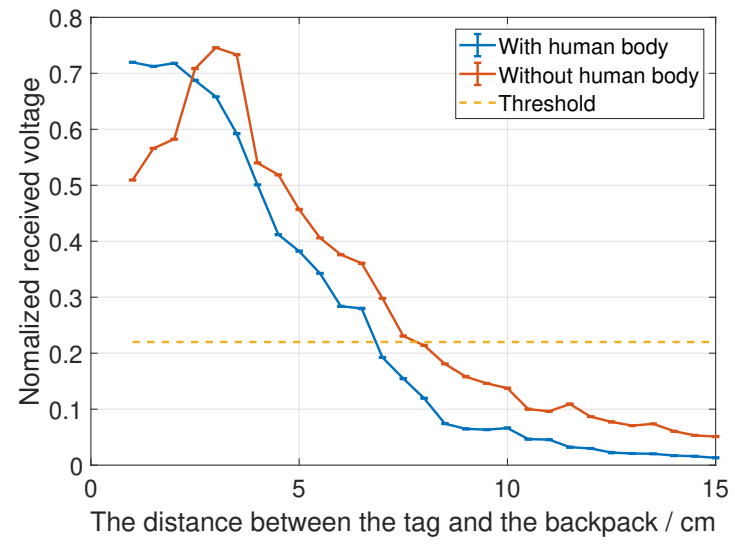

Fig. 16: The distance between the tag and the backpack with/without human body.

\subsection{Impact of Multipath}

In commercial scenarios, when the attacker and the victim are standing in the same line, there would be complex multipath due to movement in the environment, like a few people passing by or other people in the line bringing mobile phones. Therefore, in order to evaluate the stability of ReCoil, we try to simulate these situations, adding more external multipath to our system.

We first deploy some iron plates around ReCoil and measure the whole distance between the reader and the NFC card. Table 2 shows that ReCoil can still has an attack distance of $48.3 \mathrm{~cm}$. Then, when we place some smartphones around the band and the backpack, ReCoil can still reach a communication distance of $48.2 \mathrm{~cm}$. When we place a set of keys around, the whole distance between the reader and the NFC card is $48 \mathrm{~cm}$. Though the metallic materials do have some impacts on ReCoil, the results show the robustness of our system.

TABLE 2: The communication distance vs. different types of interference

\begin{tabular}{ccccc}
\hline Interference & $\begin{array}{c}\text { No } \\
\text { Interference }\end{array}$ & $\begin{array}{c}\text { Iron } \\
\text { plates }\end{array}$ & Smartphones & Keys \\
\hline $\begin{array}{c}\text { Communication } \\
\text { distance }(\mathrm{cm})\end{array}$ & 48.5 & 48.3 & 48.2 & 48 \\
\hline
\end{tabular}

Based on the results above, we obtain the good performance on accessing the victim's NFC card. However, the people who carry some metallic objects would bring about some degree of interference and affect the power that received by the hacked cards. In order to mitigate the impact of interference from the metallic objects, we evaluate if the Ferrite board can lessen the interference. We put a piece of Ferrite board on the attacker's body before she wears the waist band on. After we use the Ferrite board, the total communication distance can be enlarged to $49.6 \mathrm{~cm}$, as Fig. 17 shows. Therefore, the Ferrite material can partly reduce the interference. We also evaluate the performance when the victim puts the card into his pocket or he just holds it by hand to observe the impact of clothing. We observe that the user's clothing does have the impact on the communication distance. When the card is in the pocket, the whole distance reduces more than $1 \mathrm{~cm}$. Further, the error is stable within $1 \mathrm{~cm}$, showing the stability of our system.

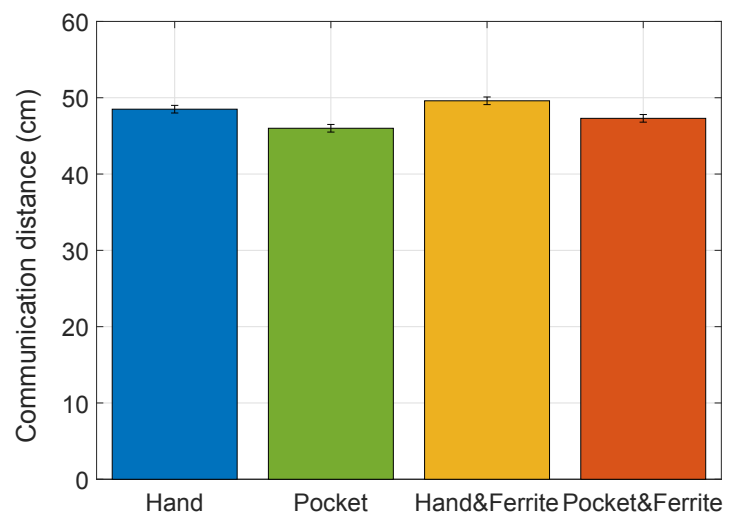

Fig. 17: The whole distance between the reader and the card when the card is in the hand/pocket with/without Ferrite board.

We also evaluate that if our system can attack cards successfully when the victim has more than one cards. Shown in Table 3, we find that the whole distance keeps decreasing with a stable error when the number of cards increases. This stems from the reason that a more-coil system divides the energy from the NFC reader into several parts, forcing a longer delay from the coils in response to the reader. As Fig. 18 shows, when the victim has two or three NFC cards, our system can still attack one of them. After extensive experiments, the results show that the three cards are randomly chosen to be attacked. In our system, if any one of the cards that the victim has is attacked, our system 
is regarded as hacking an NFC card successfully, i.e., ReCoil can still hack cards when there are collisions happening.

TABLE 3: The communication distance vs. multiple cards

\begin{tabular}{cccc}
\hline $\begin{array}{c}\text { Number of } \\
\text { cards }\end{array}$ & one & two & three \\
\hline $\begin{array}{c}\text { Communication } \\
\text { distance }(\mathrm{cm})\end{array}$ & 48.5 & 45.6 & 40.5 \\
\hline
\end{tabular}

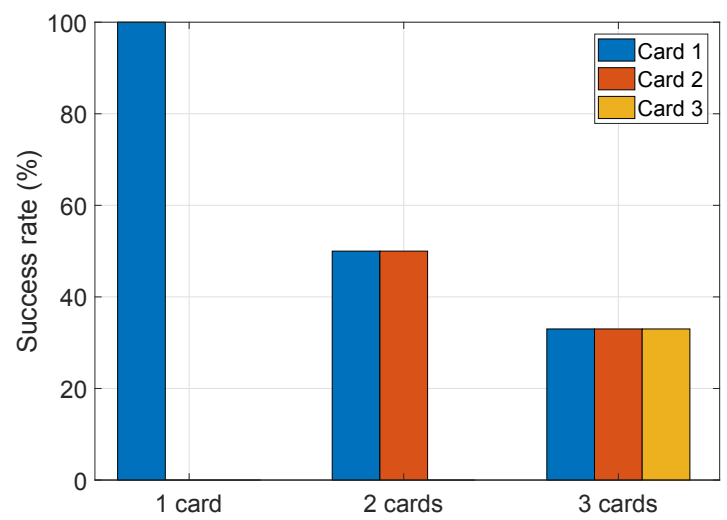

Fig. 18: The success rate of different card when there are multiple cards in the system.

TABLE 4: The communication distance vs. different smartphones

\begin{tabular}{cccc}
\hline Smartphone & $\begin{array}{c}\text { HUAWEI } \\
\text { Mate9 }\end{array}$ & Xiaomi6 & $\begin{array}{c}\text { SAMSUNG Galaxy } \\
\text { A9 Star }\end{array}$ \\
\hline $\begin{array}{c}\text { Communication } \\
\text { distance }(\mathrm{cm})\end{array}$ & 40 & 42 & 48 \\
\hline
\end{tabular}

\subsection{Case Study}

We also use different types of commercial smartphones to act as commercial readers and read NFC cards. As Table 4 shows, we use HUAWEI Mate9, Xiaomi6, SAMSUNG Galaxy A9 Star to evaluate the whole communication distance from these readers and the NFC card. The communication distance between the readers and the card in our system can still be over than $40 \mathrm{~cm}$, which validates that ReCoil can be effectively used in commercial scenarios.

\section{Discussions}

Though ReCoil coils are designed to be relatively inflexible, minute shape changes of the array can cause the resonant frequency to easily change, impacting the performance of the relays. In addition, our system can be affected by allmetal objects, like mobile phones or keys. Besides, the commercial readers or the passive tags should be opposite to one of the coils, ensuring as much magnetic flux as possible passes through the coil, and the tag receive the largest power. Thus in this section, we explain our limitations and future work.
Reducing the interference. Though the evaluation shows the robustness of our system, the system can still experience interference that absorbs the magnetic energy. Once the people around the attacker carry some metal objects, it will slightly effect the whole performance. Furthermore, when we put a smartphone face the last coil in the backpack or face the commercial reader oppositely, the NFC card behind the smartphone cannot be activated anymore. Therefore, if the victim places a mobile phone and an NFC card together when he stands in the line, our system cannot attack the card anymore. At this time, the attacker needs to find a new victim. Thus, how to relieve the interference from smartphones or how to hack the NFC-based smartphones directly would be potential problems in the future.

Improving the robustness. As shown in Sec.5 and Sec.7. the NFC cards in our system should totally face the last coil in the backpack, because the cards can largely receive the magnetic power under the circumstances. We find that there is a tradeoff between the width and the length of the attacking area. Since we need to use the small square coil to focus the magnetic field to a certain direction, the range of attacking width is very limited. However, we have no prior knowledge on the location of the victim, weakening our success rate of attack. We will consider algorithms of blind search in the future to make our system more sustainable.

Resolving the collisions. Though our system can attack the NFC cards when the victim has three cards or more, the attacking distance is undermined when more NFC cards are added to ReCoil. The several cards suffer magnetic interference when they are put together, resulting in limited power for them to wake up. Collision resolution involves the optimization of anti-collision protocol, thus, using USRP to operate NFC protocols and optimizing the anti-collision mechanism should be essential to solve this problem.

\section{Conclusion}

In this paper, we design the system, ReCoil to attack the NFC cards by using passive relays. Unlike prior work, which required power consumption, our approach is battery-free and effortless for the attacker to wear. Instead of modifying the protocol of NFC readers, we transmit the signal of commercial NFC readers far away by passive relays, making our system stealthy and hard to be located. After designing the algorithm to search the optimal coil parameters, we build an MCR-WPT system. The waist band and the three-coil box put into the backpack can boost the communication range of NFC hacking. We attach tunable capacitors on these coils to match the unique inductance of different coils. Meanwhile, we use Ferrite board to reduce interference. Through extensive experiments, the farthest distance ReCoil can achieve is $49.6 \mathrm{~cm}$, which is as much as ten times the communication distance of the existing NFC system. ReCoil is compatible with commercial smartphones, achieving a distance over $40 \mathrm{~cm}$. Further, when the victim has several NFC cards together, ReCoil can still randomly select one and attack it successfully.

\section{REFERENCES}

[1] R. Nelson, "Red stands out at europes emv 2017," Working at the cutting edge of technology, p. 18, 2017. 
[2] I. O. for Standardization. (2018) Iso/iec 14443-4:2018. [Online]. Available: https://www.iso.org/standard/73599.html

[3] M. RAHMAN and H. ELMILIGI, "Classification and analysis of security attacks in near field communication," International Journal of Business and Cyber Security, vol. 1, no. 2, 2017.

[4] F. Dang, P. Zhou, Z. Li, E. Zhai, A. Mohaisen, Q. Wen, and M. Li, "Large-scale invisible attack on afc systems with nfc-equipped smartphones," in proceedings of International Conference on Computer Communications. IEEE, 2017, pp. 1-9.

[5] C. Bermejo and P. Hui. Steal your life using 5 cents: Hacking android smartphones with nfe tags.

[6] J. B. Hur and J. A. Shamsi, "A survey on security issues, vulnerabilities and attacks in android based smartphone," in proceedings of International Conference on Information and Communication Technologies (ICICT). IEEE, 2017, pp. 40-46.

[7] J. Jumić and M. Vuković, "Analysis of credit card attacks using the nfc technology," in proceedings of International Convention on Information and Communication Technology, Electronics and Microelectronics (MIPRO). IEEE, 2017, pp. 1251-1255.

[8] L. Francis, G. Hancke, K. Mayes, and K. Markantonakis, "Practical nfc peer-to-peer relay attack using mobile phones," in proceedings of International Workshop on Radio Frequency Identification: Security and Privacy Issues. Springer, 2010, pp. 35-49.

[9] K. Markantonakis, L. Francis, G. Hancke, and K. Mayes, "Practical relay attack on contactless transactions by using nfc mobile phones," Radio Frequency Identification System Security: RFIDsec, vol. 12, p. 21, 2012

[10] Z. Wang, Z. Xu, W. Xin, and Z. Chen, "Implementation and analysis of a practical nfc relay attack example," in proceedings of International Conference on Instrumentation, Measurement, Computer, Communication and Control (IMCCC). IEEE, 2012, pp. 143-146.

[11] T. Korak and M. Hutter, "On the power of active relay attacks using custom-made proxies," in IEEE International Conference on RFID. IEEE, 2014, pp. 126-133.

[12] Z. Kfir and A. Wool, Picking virtual pockets using relay attacks on contactless smartcard. IEEE, 2005.

[13] E. Lee, "Nfc hacking: The easy way," in Defcon hacking conference, vol. 20, 2012, pp. 63-74.

[14] M. Mehrnezhad, M. A. Ali, F. Hao, and A. van Moorsel, "Nfc payment spy: a privacy attack on contactless payments," in International Conference on Research in Security Standardisation. Springer, 2016, pp. 92-111.

[15] Y.-J. Tu and S. Piramuthu, "On addressing rfid/nfc-based relay attacks: An overview," Decision Support Systems, p. 113194, 2019.

[16] (2018) Long range nfc detection. [Online]. Available: https: //www.youtube.com/watch? $\mathrm{v}=\mathrm{Wwy} 8 \mathrm{ButHbcU}$

[17] H. Kortvedt and S. Mjolsnes, "Eavesdropping near field communication," in The Norwegian Information Security Conference (NISK), vol. 27, 2009, p. 5768.

[18] Z. Wang, "Information security vulnerabilities of nfc technology and improvement programs," in Proceedings of the 2018 International Conference on Information Science and System. ACM, 2018, pp. 196-199.

[19] T. W. Brown, T. Diakos, and J. A. Briffa, "Evaluating the eavesdropping range of varying magnetic field strengths in nfc standards," in European Conference on Antennas and Propagation (EuCAP). IEEE, 2013, pp. 3525-3528.

[20] I. Kirschenbaum and A. Wool, "How to build a low-cost, extended-range rfid skimmer." in USENIX Security Symposium, vol. 4, 2006.

[21] B. Gulbahar, "A communication theoretical analysis of multipleaccess channel capacity in magneto-inductive wireless networks," IEEE Transactions on Communications, vol. 65, no. 6, pp. 2594-2607, 2017.

[22] G. Dumphart, E. Slottke, and A. Wittneben, "Magneto-inductive passive relaying in arbitrarily arranged networks," in Communications (ICC), 2017 IEEE International Conference on. IEEE, 2017, pp. $1-6$.

[23] N. Ahmed, "Magneto inductive communication system for underwater wireless sensor networks," 2017.

[24] A. Vallecchi, S. Chu, L. Solymar, C. J. Stevens, and E. Shamonina, "Coupling between coils in the presence of conducting medium," IET Microwaves, Antennas and Propagation, 2018.

[25] S. Kisseleff, I. F. Akyildiz, and W. H. Gerstacker, "Throughput of the magnetic induction based wireless underground sensor networks: Key optimization techniques," IEEE Transactions on Communications, vol. 62, no. 12, pp. 4426-4439, 2014.
[26] Z. Sun and I. F. Akyildiz, "On capacity of magnetic inductionbased wireless underground sensor networks," in Proceedings of International Conference on Computer Communications. IEEE, 2012, pp. $370-378$.

[27] J. Jadidian and D. Katabi, "Magnetic mimo: How to charge your phone in your pocket," in Proceedings of the 20th annual international conference on Mobile computing and networking. ACM, 2014, pp. 495-506.

[28] L. Shi, Z. Kabelac, D. Katabi, and D. Perreault, "Wireless power hotspot that charges all of your devices," in Proceedings of the 21st Annual International Conference on Mobile Computing and Networking. ACM, 2015, pp. 2-13.

[29] J. Reid, J. M. G. Nieto, T. Tang, and B. Senadji, “Detecting relay attacks with timing-based protocols," in Proceedings of the 2nd ACM symposium on Information, computer and communications security. ACM, 2007, pp. 204-213.

[30] Y. Zhuang, A. Yang, G. P. Hancke, D. S. Wong, and G. Yang, "Energy-efficient distance-bounding with residual charge computation," IEEE Transactions on Emerging Topics in Computing, 2017.

[31] H. Kılınç and S. Vaudenay, "Contactless access control based on distance bounding," in International Conference on Information Security. Springer, 2017, pp. 195-213.

[32] T. Halevi, D. Ma, N. Saxena, and T. Xiang, "Secure proximity detection for nfc devices based on ambient sensor data," in European Symposium on Research in Computer Security. Springer, 2012, pp. 379-396.

[33] I. Gurulian, C. Shepherd, E. Frank, K. Markantonakis, R. N. Akram, and K. Mayes, "On the effectiveness of ambient sensing for detecting nfc relay attacks," in Trustcom/BigDataSE/ICESS, 2017 IEEE. IEEE, 2017, pp. 41-49.

[34] C. Shepherd, I. Gurulian, E. Frank, K. Markantonakis, R. N Akram, E. Panaousis, and K. Mayes, "The applicability of ambient sensors as proximity evidence for nfc transactions," in Security and Privacy Workshops (SPW). IEEE, 2017, pp. 179-188.

[35] S. Hameed, B. Hameed, S. A. Hussain, and W. Khalid, "Lightweight security middleware to detect malicious content in nfc tags or smart posters," in Proceedings of International Conference on Trust, Security and Privacy in Computing and Communications (TrustCom). IEEE, 2014, pp. 900-905.

[36] D. M. Parmar and B. Panchal, "Securing nfc againts data corruption and eavesdropping using diffie hellman," 2017.

[37] W. Shen, H. Wu, H. Xu, and P. Li, "A new middleware architecture for rfid data management," in International Conference on Emerging Internetworking, Data \& Web Technologies. Springer, 2017, pp. 212221.

[38] I. Gurulian, R. N. Akram, K. Markantonakis, and K. Mayes, "Preventing relay attacks in mobile transactions using infrared light," in Proceedings of the Symposium on Applied Computing. ACM, 2017, pp. 1724-1731.

[39] ST25. (2016) How to design a $13.56 \mathrm{mhz}$ customized antenna for st25 nfc / rfid tags. [Online]. Available: https://www.st.com/content/ccc/ resource/technical/document/application_note/d9/29/ad/ cc/04/7c/4c/1e/CD00221490.pdt/tiles/CD00221490.pdt/jcr: content/translations/en.CD00221490.pdt

[40] Y. Cheng and Y. Shu, "A new analytical calculation of the mutual inductance of the coaxial spiral rectangular coils," IEEE Transactions on Magnetics, vol. 50, no. 4, pp. 1-6, 2013.

[41] A. Anele, Y. Hamam, L. Chassagne, J. Linares, Y. Alayli, and K. Djouani, "Computation of the mutual inductance between aircored coils of wireless power transformer," in Journal of Physics: Conference Series, vol. 633, no. 1. IOP Publishing, 2015, p. 012011.

[42] H. Tavakkoli, E. Abbaspour-Sani, A. Khalilzadegan, G. Rezazadeh, and A. Khoei, "Analytical study of mutual inductance of hexagonal and octagonal spiral planer coils," Sensors and Actuators A: Physical, vol. 247, pp. 53-64, 2016.

[43] F. Liu, Y. Yang, D. Jiang, X. Ruan, and X. Chen, "Modeling and optimization of magnetically coupled resonant wireless power transfer system with varying spatial scales," IEEE Transactions on Power Electronics, vol. 32, no. 4, pp. 3240-3250, 2016.

[44] H. Dai, Y. Liu, G. Chen, X. Wu, T. He, A. X. Liu, and H. Ma, "Safe charging for wireless power transfer," IEEE/ACM Transactions on Networking (TON), vol. 25, no. 6, pp. 3531-3544, 2017.

[45] B. Clerckx and E. Bayguzina, "Waveform design for wireless power transfer," IEEE Transactions on Signal Processing, vol. 64, no. 23, pp. 6313-6328, 2016. 
[46] J. Dai and D. C. Ludois, "A survey of wireless power transfer and a critical comparison of inductive and capacitive coupling for small gap applications," IEEE Transactions on Power Electronics, vol. 30, no. 11, pp. 6017-6029, 2015.

[47] S. Y. R. Hui, W. Zhong, and C. K. Lee, "A critical review of recent progress in mid-range wireless power transfer," IEEE Transactions on Power Electronics, vol. 29, no. 9, pp. 4500-4511, 2013.

[48] M. Dorigo and T. Stützle, "Ant colony optimization: overview and recent advances," in Handbook of metaheuristics. S Springer, 2019, pp. 311-351. 